\title{
Helium and Oxygen Abundances in SMC Planetary Nebulae
}

\author{
R. D. D. Costa, J. A. de Freitas Pacheco and T. P. Idiart \\ Instituto Astronômico e Geofísico/USP, Av. Miguel Stéfano 4200, \\ 04301-904 - São Paulo - SP, Brazil
}

\begin{abstract}
In this work we report new high quality spectroscopic data for a sample of $\mathrm{PNe}$ in the SMC, aiming to derive physical parameters and chemical abundances, in particular to settle the question concerning the oxygen discrepancy found for type I planetaries with respect to stars and HII regions.
\end{abstract}

\section{Introduction}

Planetary nebulae can provide valuable clues to understand the evolution of stellar systems like the Magellanic Clouds. In particular, they give informations on intermediate mass stars from which they are originated. While helium and nitrogen abundances are affected by dredge-up episodes along the progenitor life, abundances of other elements like oxygen, neon and argon trace the chemical history of the interstellar medium. Low mass (old) stars are supposed to suffer a low contamination from the first dredge-up, and these objects have been used by several authors to estimate the primordial He abundance.

In this work we report new data for a sample of $18 \mathrm{PNe}$ in the SMC, and we show that there is an anti-correlation between the nitrogen enrichment and the oxygen abundance (supposed to represent the arrow of time). Combining our previous LMC data with existing data in the literature, we found a similar anti-correlation, also observed for galactic type-I PNe.

Face to these unexpected results, the use of $\mathrm{He} / \mathrm{H}$ vs $\mathrm{O} / \mathrm{H}$ diagrams for low mass planetaries, aiming to derive primordial He values, should be taken cautiously.

\section{Observations and Data Reduction}

Observations for this work were made with two different telescopes and instrumental configurations: LNA $1.60 \mathrm{~m}$ telescope, in Brazil, using a Boller \& Chivens Cassegrain spectrograph, grating of 300 lines $/ \mathrm{mm}$, allowing a $4.4 \AA /$ pixel dispersion in an observational run from 18 to 21/Jul/1999, and ESO-Chile 1.52 $\mathrm{m}$ telescope, with a Boller \& Chivens Cassegrain spectrograph, grating of 400 $\mathrm{l} / \mathrm{mm}$ allowing a $2.2 \AA /$ pixel dispersion, in an observational run from 15 to 20/Aug/1999.

Line fluxes were calculated by gaussian profiles and reddening was derived from the Balmer $\mathrm{H} \alpha / \mathrm{H} \beta$ ratio. Electron density was calculated from [SII] 
$6716 / 6730$ line ratio and electron temperatures from [OIII] and [NII] lines. Ionic abundances were derived from a 3-level atom model and then Elemental abundances were calculated through IC-factors. Typical uncertainties in abundances are of 0.1 to 0.2 dex.

\section{Discussion}

From our sample we derive the following mean abundances:

$$
\begin{aligned}
& \mathrm{He} / \mathrm{H}=0.113 \pm 0.019 \\
& \varepsilon(\mathrm{O})=8.22 \pm 0.24 \\
& \varepsilon(\mathrm{N})=7.11 \pm 0.44 \\
& \varepsilon(\mathrm{Ne})=7.47 \pm 0.23 \\
& \varepsilon(\mathrm{S})=6.34 \pm 0.18 \\
& \varepsilon(\mathrm{Ar})=5.79 \pm 0.28
\end{aligned}
$$

These values for SMC planetary nebulae are compatible with those from their galactic counterparts, however, to classify the objects among the Peimbert (1983) types, we took into account the smaller enrichment of the SMC interstellar medium with respect the Galaxy, as already made to the LMC (see de Freitas Pacheco et al. 1993 for further details). Following this scheme,we classified 5 objects as type-I PNe.

Abundances from our sample correlate as expected: helium vs. $\log (\mathrm{N} / \mathrm{O})$ reflect the mass spectrum of the progenitors, with objects of higher masses, which experienced more dredge-up episodes, displaying higher abundances. Oxygen and neon, elements not produced by the progenitors have also correlated abudances, reflecting the interstellar medium at the moment of each progenitor's formation.

A clear anti-correlation appeared from our data, between $\varepsilon(\mathrm{O})$ and $\log (\mathrm{N} / \mathrm{O})$ values, indicating that in the timescale of intermediate mass stars evolution in the Magellanic Clouds, oxygen abundance cannot be seen as a steadily increasing function of time. Two possible interpertations arise to explain this behavior: oxygen conversion into nitrogen, or a relationship between the efficiency of the dredge-up process with the metallicity of the progenitors. As a consequence, one shoud be cautious when classifying extragalactic planetaries on the basis of chemical abundances only. These problems are examined in more detail by Costa et al. (2000).

\section{References}

Costa, R.D.D., de Freitas Pacheco, J.A. \& Idiart, T.P. 2000, A\&A, submitted de Freitas Pacheco, J.A., Costa, R.D.D. Barbuy, B. \& Idiart, T.P. 1993, A\&A 271,429

Peimbert,M. in IAU Symposium 103, Planetary Nebulae, ed. R.D. Flower (Dordrecht: Reidel), 233 